\title{
Electronic Repair Concepts for Long-Duration Spaceflight
}

\author{
John Easton ${ }^{\dagger}$ and Richard D. Pettegrew ${ }^{\dagger}$. \\ National Center for Space Exploration Research ${ }^{\dagger}$, Cleveland, Ohio, 44135 \\ and \\ Peter M. Struk \\ NASA Glenn Research Center, Cleveland, Ohio, 44135
}

\begin{abstract}
[Abstract] Constraints on the mass and volume that can be allocated for electronics spares and repair equipment on long-duration space missions mean that NASA must look at repair strategies beyond the traditional approach, which has been to replace faulty subsystems in a modular form, termed Orbital Replacement Units or Line Replacement Units. Other possible strategies include component and board-level replacement, modular designs that allow reprogramming of less-critical systems to take the place of more critical failed systems, and a blended approach which uses elements of each of these approaches, along with a limited number of Line Replacement Units. This paper presents some of the constraints and considerations that affect the decision on how to approach electronics repair for long duration space missions, and discusses the benefits and limitations of each of the previously mentioned strategies.
\end{abstract}

\section{Introduction}

$\mathrm{N}$ ASA's plans for long duration missions to the Moon and, especially, to Mars require a much greater degree of self sufficiency on the part of the crew than ever before. Such missions will have greatly reduced logistic support from Earth, compared to International Space Station (ISS) operations. Returning to Earth in the event of an emergency may not be an option either, as a lunar return flight could require two to three days, and a Martian return flight will require much longer.

One area of mission support that NASA must plan for is electronic repairs. Despite the rigorous testing required by NASA, electronics faults have already occurred in both Space Shuttle and ISS operations ${ }^{1,2}$, leading to the use of backup systems or loss of capability to some degree. While the electronics and other systems used in a long duration space mission will undergo rigorous testing, the crew of such missions will most likely encounter an electronics failure at some point in the mission. With the design of the Crew Exploration Vehicle (CEV) already beginning and likely influencing the design of future spacecraft and hardware, it is not too early for NASA to begin exploring and designing techniques and tools for crew members conducting electronics repair during long duration space mission. These considerations include system design (for accessibility, parts type and sizes, and board complexity), repair infrastructure (including diagnostic capabilities, tools, and other needed equipment), and logistics constraints. The decisions on how to approach each of these considerations depends on the overall repair strategy that is chosen.

The current repair scheme used for the ISS focuses on orbital replacement units (ORU). An ORU is a modular electronics assembly or system, designed to be replaced as a unit. When an electronic fault is detected, it is isolated to specific ORU through system performance information. The crew then removes the entire ORU, replacing it with a spare. Most replacement ORUs are flown from the ground as needed, with limited spares on hand. Preliminary designs for the CEV use a similar design for electronic systems, also referred to as line replacement units $\left(\mathrm{LRU}^{* *}\right)^{3}$.

Previous work regarding electronics repairs during missions has focused largely on the economics of performing repairs, in terms of crew time, upmass, and volume of spares. Accola et. al. ${ }^{4}$ examined the implications of crew members performing varying amounts of repair onboard the then-proposed Space Station Freedom. Their model

\footnotetext{
${ }^{\dagger}$ Staff Scientist, NCSER, 21000 Brookpark Rd, MS 110-3, AIAA Member

* Aerospace Engineer, NASA GRC, 21000 Brookpark Rd, MS 110-3, AIAA Member

${ }^{* *}$ This paper uses the LRU name convention for any modular electronics system onboard a spacecraft.
} 
predicted that increasing crew performed repairs could save on mass and volume of spares compared to reduced levels of repair, up to eliminating repairs altogether and simply replacing faulty LRUs with good ones. These repairs cost a small amount of crew time, with this cost more than offset by volume and mass considerations.

NASA researchers have also begun studies of soldering electronics components in reduced gravity. These studies include a demonstration of soldering wires in orbit ${ }^{5}$ as well as many studies of soldering aboard a reduced gravity aircraft laboratory $6,7,9,10$. These later studies show an increase in solder joint void fraction in reduced gravity compared to solder joints formed in normal gravity. An increase in joint void fraction indicates a greater risk for the joint to fail over time; these results show a need for NASA to explore methods of mitigating the increase in void fraction.

This paper will discuss the factors that must be considered when choosing a repair strategy for long-duration space missions, and several potential repair strategies are examined. The relative advantages and disadvantages of each approach are discussed, as well as suggestions for a 'blended' approach, in which elements of each may be the optimal solution.

\section{Factors Affecting Electronics Repair During Space Missions}

A number of factors must be considered when selecting a strategy for electronics repair during a mission, particularly since each of these factors needs to be addressed during the design of the spacecraft and the mission profile. For the purpose of this paper, these factors are defined as Electronic System Design (which includes design considerations at the board level and subsystem level), Diagnostics, Repair Infrastructure (which includes the tools and equipment needed to perform the repairs, as well as the logistics of the spares stores) and Crew Considerations. While the details on each of these topics are beyond the scope of this paper, each will be discussed in a high-level fashion in the following subsections.

\section{A. Electronic System Design}

The design of the electronics for a long-duration mission is one of the first factors to be considered, since these designs can enable or prevent elements of the different repair strategies, and decisions regarding the electronic systems design are likely to be made early in the overall spacecraft design process. With regard to repair considerations, electronic systems must first be designed for accessibility, meaning the crew member must have access to the system in order to repair it. While this may seem self-evident, volume and other design constraints may make a significant portion of the subsystems inaccessible to crew members from inside the craft. If this is the case, then either robotic or EVA (Extra Vehicular Activity) provisions must be made to allow access.

If the selected repair strategy involves component-level (or board-level) repair, then the concept of accessibility
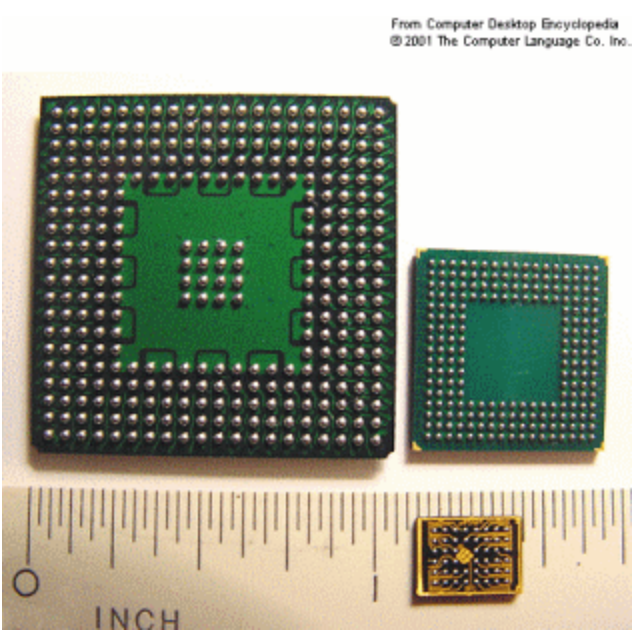

Figure 1: Example of ball-grid array (BGA) electronic component, showing the array of contacts on the bottom side of the component. needs to be extended down several levels further. Individual boards (within the LRU or subsystem) must be accessed and disconnected, and, if components are to be replaced, then the conformal coating on the board must be removable. While most coatings can be removed with various methods in ground-based facilities, limitations on chemicals and equipment that can be flown make conformal coating removal an accessibility issue that must be considered.

The strategy of component-level repair, as well as the degree to which that strategy is implemented will also require consideration in the design of circuit boards and the choice of components used. If manually-operated repair tools (such as a soldering iron) are used, limits will exist on what components can be repaired. One example of such a component is the 'ball grid array' configuration of an integrated circuit (Figure 1), in which the leads are not accessible to a soldering iron. Other factors, such as the pitch of the leads, may also make manual repairs intractable in some cases. While some of these limitations may be addressed using other repair technologies (such as a semi-automated repair station), such limitations may be minimized or avoided through careful design. 


\section{B. Diagnostics}

Regardless of the repair philosophy chosen, the problem remains that diagnostics must be included to determine where the problem lies in a faulty system. At a high level, the first layer of diagnostics will include isolation of the problem to a specific subsystem, or LRU. This may be through symptomatic diagnosis, or through system-wide levels of built-in tests (BIT).

If LRU replacement is chosen, this level of diagnostics is sufficient. If component-level repair is to be implemented, then further diagnostics are needed to pinpoint which component is faulty. A trade study completed by the authors ${ }^{11}$ determined that diagnostics needed for component-level repair are likely to include non-functional test equipment, which tests components in a non-powered state and compares their signatures against known good data. These diagnostics will also be needed for post-repair verification, prior to returning a subsystem to service. Additionally, crew members will require standard diagnostic tools such as oscilloscopes, multimeters, etc., to properly locate a faulty component. Diagnostic capabilities also require some levels of crew training, and may also include a ground support via telemetry.

\section{Repair Infrastructure Requirements}

Within the scope of this paper, repair infrastructure is defined as the set of tools and spare parts required to implement the repair. The tools requirements will vary, depending on the strategy chosen. Most strategies are likely to entail at least a limited set of small hand tools, for disconnecting fasteners, etc. Component-level repair will also require some specialized tools, such as

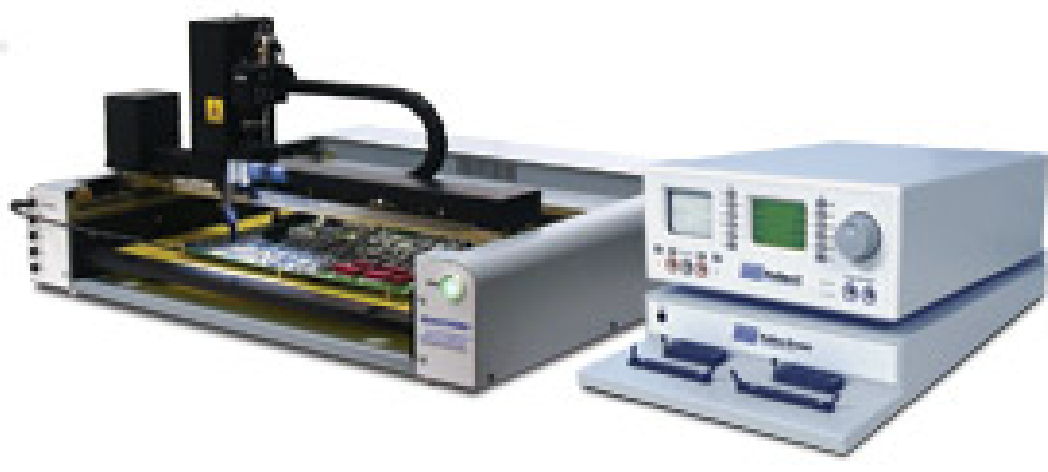

Figure 2: Example of a semi-automated, electronics diagnostic system with $X-Y$ translation stage. a soldering iron or other soldering technology, and specialized tools for component removal. If a semiautomated component-level repair is implemented, then an $\mathrm{X}-\mathrm{Y}$ positioning stage (Figure 2) is also needed.

The logistical aspect of spares represents a key element of the infrastructure requirement, with wide variations between the requirements for LRU replacement and componentlevel repair. Careful choices must be made to insure that adequate quantities of spares are available, in the event of multiple failures. The issue of mass and volume required to provide that number of spares is likely to be a central issue when deciding on a repair strategy.

\section{Crew Considerations}

An additional factor in the selection of a repair strategy is the skill and training requirements on the crew, as well as time required to implement the various repair strategies. Each of the different repair strategies will require significantly different specific skills and training to implement the repairs, although some of the skill requirements may be partially mitigated through the use of a 'tele-science' approach, in which specific portions of the diagnosis of the problem are handled in conjunction with ground-based engineers. Up-linked training materials on specific repair issues can also help minimize the need to pre-train the crew for every specific incident, though a baseline of knowledge and experience will clearly be needed.

Crew time required to perform a repair will also be a relevant consideration. Critical systems may (in some circumstances) require repair strategies which can be implemented very quickly, while other repairs may not be time-critical. Crew time is always considered a high-value resource, but the value of the crew time must be weighed against all of the other considerations when examining the choice of repair strategy. Clearly, less crew time is required for LRU replacement, but this may be overridden by the need to reduce the volume of spares for the mission. 


\section{Possible Strategies for Electronics Repair}

The general strategies for addressing electronic failures during a mission can be classified broadly as:

1) LRU Replacement: This approach involves removing and replacing modular subsystems, which are comprised of 'blocks' of circuit boards.

2) Component-Level Repair: This approach entails repair of individual faulty components on a circuit board

3) System Re-Programming / "Scavenging": This approach involves the use of "common modules" (to the degree possible) which can be removed from service on less crucial systems, and re-programmed to replace critical systems that have undergone failure.

4) Combined Approach: This approach uses a blended combination of each of the three distinct strategies, in an attempt to provide an optimal repair/maintenance solution.

Additionally, the 'component-level repair' strategy can be divided into manually performed repairs (i.e., hand tools only), and semi-automated repair (with mechanized aids for diagnostics, component placement, and soldering). Each of these approaches has distinct advantages and disadvantages, in terms of system design requirements, the diagnostics and repair infrastructure needed, and crew considerations. The following sections examine some possible implementations of these strategies, with discussion of the benefits and limitations of each approach.

\section{LRU Replacement}

LRU's are modular subassemblies that generally contain a series of related circuit boards, designed to collectively perform a specific function (or multiple functions) within a larger system. These units are packaged for relatively easy installation and removal, and require comparatively low crew time and training to use.

A representative range in LRU spatial dimensions is from about 3" x 3" x 3" to 28” x 17" x 7", with mass ranging from approximately $1 \mathrm{lb}$ to $75 \mathrm{lbs}$. Figure 3 shows an example of a typical LRU ${ }^{12}$. When an electronic system fails, the fault is isolated to a specific LRU, using system performance observations and some level of builtin diagnostics. The crew member then removes the entire LRU and replaces it with a spare or relies on a backup system. The faulty LRU then returns to Earth with

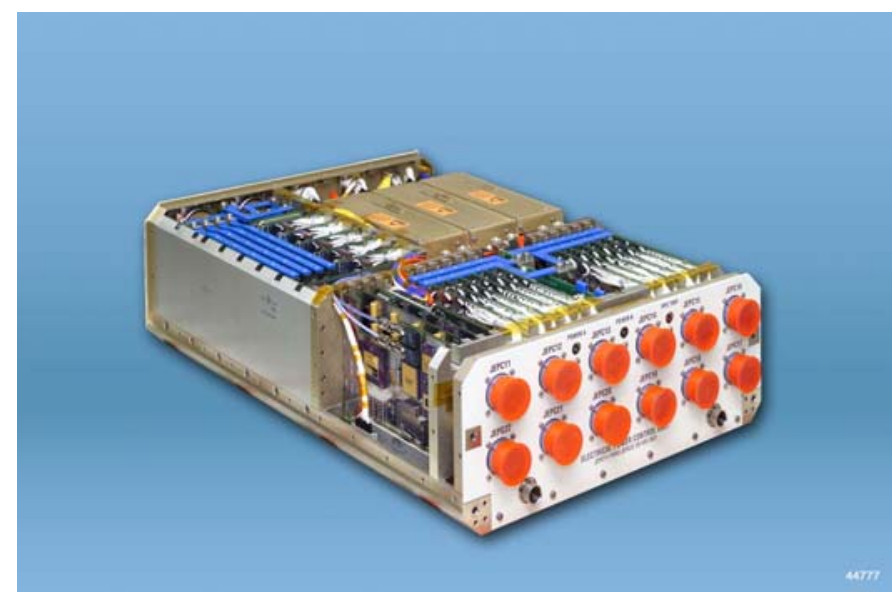

Figure 3: Example of an LRU (Electrical Power and Control Unit, referred to as EPCU) ${ }^{12}$. the shuttle, where it may be repaired, tested and returned to a pool of spares awaiting return to space.

The use of LRU's affords several important benefits. Since the unit can be thoroughly tested prior to flight, the reliability of LRU replacement as a repair strategy is very high. Also, the requirement on crew training and experience is minimal, and the LRU's can generally be replaced fairly quickly and with minimal tools.

However, the LRU replacement strategy carries a high penalty in terms of mass and volume, considering the likely requirements of multiple spares per system. Furthermore, long-duration missions may require travel times that prohibit resupply from Earth, particularly Martian missions. The mission must bring along all potentially-needed spares with them. Given the complexity of a long duration spacecraft, the number of spares required for adequate mission safety for each LRU is probably prohibitive.

\section{Component-Level Repair}

While LRU replacement approaches electronic repair from the standpoint of subsystem replacement, componentlevel repair addresses the problem at the lowest possible level. This approach offers the greatest savings in terms of mass and volume of spares, it does require at least some additional tools and infrastructure (such as additional 
diagnostics, beyond the BIT diagnostics already used in LRU's). Further, crew training becomes much more important, and time requirements to perform the repairs increase.

NASA has already begun investigation of component-level electronics repair, with the CLEAR project (standing for Component-Level Electronic-Assembly Repair) ${ }^{13}$. This effort, which is a combination of trade studies, normal gravity demonstrations, and reduced gravity testing aboard aircraft facilities and the ISS, is examining the feasibility of manually-operated, component-level repairs (i.e., with a soldering iron) for long-duration missions. Early efforts have shown the general feasibility of electronics repair in this environment, building on previous research done on the effects of the reduced gravity environment on the soldering process ${ }^{6-10}$.

An extension of that work would be to perform component-level repair using a semi-automated re-work station, as previously noted. Such stations use computer controlled translation stages and machine vision to position diagnostics or soldering/de-soldering devices on faulty components whose size or configuration would not typically permit manual repair. This capability would allow repair of a broader class of electronic components, as well as advanced soldering technologies such as infrared re-flow, or convective heating re-flow. Systems of this type would allow inclusion of more components in the "repairable" category than manual repair (thereby further reducing the need for spare LRU's), and would help to minimize the need for crew experience in manual repairs.

\section{System Re-Programming / Scavenging}

The notion of re-programming or scavenging systems to repair or work around failures is a strategy that could have several embodiments. One version of this involves the use of existing, reprogrammable resources to reprogram faulty subsystems and work around damaged components. Although there are several methods to implement this, field programmable gate array (FPGA) devices ${ }^{* * *}$ can be used as an example. With an FPGA, the actual configuration is typically loaded into the FPGA after power-up. The configuration can be stored in non-volatile memory and could be changed. For example, if the failed subsystem were a power supply or power conditioning module, re-programming could allow supply voltages to be programmed to different settings. The same approach can be taken at the LRU level, where sub-systems could be comprised of items that could be transferred to different applications. This could include computer cards, motion amplifiers/controllers, etc., with the different functionality being determined by the FPGA code that is loaded.

Another strategy for recovering from electronic failures would be to scavenge existing parts or subsystems to repair a faulty circuit board, or work around a fault. With scavenging techniques, crew members would remove components from a good board in another system deemed less crucial to operations and crew well-being than the system with the damaged circuit. Crew skill and time requirements for this approach are not very different from the component-level repair plan already discussed. This strategy, though, will require considerable work prior to launch. Mission designers and planners will have to compile a list of components available that is easily accessible to the crew or ground support teams, use common components across all circuit boards to the greatest degree possible, and prioritize systems and circuit boards to aid in deciding which circuit board to scavenge.

\section{Combined Approach}

The discussions of the strategies listed in this paper may seem to suggest that these strategies are exclusive of each other, when the most practical approach is likely to be a combination of each of these. For example, a comparatively small number of spare LRU's may be required for critical systems, so that the functional system could be brought back on-line while the faulty component is found and repaired in the original unit. Less critical systems, in certain areas, may be addressed using a scavenging and re-programming approach, with the faulty LRU being repaired, then re-installed in place of the scavenged LRU. An additional implementation could involve boardlevel replacement within the LRU for components that are not easily repairable given the infrastructure or tools that are available. This approach is a compromise between true component-level repair and full LRU replacement; while it does require more spare storage space than true component-level repair, it requires considerably less space than that of full LRU replacements. A combined strategy of component-level repair on some (significant) fraction of parts, with non-repairable parts being replaced at the circuit-card level, along with a minimal number of LRU spares, and LRU's that are designed (where possible) for re-programming and re-use, would allow the greatest flexibility in repairing a range of faults while still minimizing the logistics footprint required, to the degree possible.

\footnotetext{
FPGA's are programmable hardware devices, in which the internal hardware configuration is controlled via software, allowing implementation of custom logic circuits.
} 


\section{Discussion}

The previously described strategies represent three distinct approaches to electronics repair on long-duration space missions. Each carries certain advantages over the others, along with corresponding disadvantages. For the purpose of this discussion, comparisons will be made in two broad areas; ease of repair / extent of crew training required, and volume and mass savings. A major assumption in this discussion is that all of the three methods are in fact practical, and can be implemented effectively from an operational standpoint. In practice, only the LRU replacement method has been previously implemented, and therefore fully proven. While early results show significant promise, component-level repair is a strategy still being investigated by NASA ${ }^{13}$.

\section{Ease of Repair and Extent of Crew Training}

The first area of comparison is ease of repair and minimizing training, repair, and practice time for the crew. Crew training and repair time is an important consideration because of the extensive amount of training for all aspects of a mission prior to launch and the planned scientific, housekeeping, and maintenance exclusive of repair during a mission. For these reasons, plans that reduce the training and repair workload for the crew are typically given strong consideration by NASA planners.

Replacing LRUs has advantages over the other repair plans in terms of required crew training time, practice time, and time spent performing a repair. Replacing a LRU requires little training beyond familiarity with electrical and mechanical connectors for the LRUs, which is required for any repair scenario. Replacement strategies can also use a standardized set of procedures, which also simplifies the repair process. Replacing LRUs also limits the amount of ground support required for the repair. Ground support teams will most likely receive BIT results regardless of the repair plan used, and need only advise crew members as to which LRU to replace, where to find the replacement and stow the faulty unit, and any specific procedures for replacing the LRU in question.

The other repair plans require more crew training and time than simply replacing the LRU. Reprogramming FPGA or similar devices requires training to open an LRU, find the faulty component, and load a new program. This also requires extensive diagnostic testing, and involvement with a ground support team to properly diagnose the fault, and to possibly send new code for the failed device from Earth. Scavenging and replacing a failed component may face many of the same ground support requirements as reprogramming components - aid in diagnosis and planning and implementing a repair. These processes also require extensive crew training prior to the flight. This training includes soldering techniques with the available tools, performing diagnostic testing and some interpretation of the results, and techniques for handling a circuit board and components beyond soldering component leads. Crew members may also require practice in soldering techniques to retain competency in performing repairs.

\section{Volume and Mass Savings}

Saving on the volume and mass of electronic repair tools and parts, as with any other system carried into space, is of critical importance. Mass and volume savings in one area translates to allowances in other areas. Repairing a board requires some volume and mass, both in tools and spare parts. This plan requires storing spare circuit boards and spare components, as well as providing diagnostic and repair tools. Scavenging and reusing or reprogramming components saves mass and volume by reducing the amount of spare circuit boards and components, but does not eliminate the need for diagnostic and repair tools. Replacing LRUs, though, is likely to require much more volume and mass, compared to the other methods. While replacement does not require the same level of diagnostic and repair tools that may be required by the other methods, it does require full-sized LRUs to be stored. Given that an entire LRU typically contains multiple boards, as well as a framework and external housing, it is clear that replacing an entire LRU is less efficient, in terms of storage of spares, than the other methods. This is particularly significant when considering the need for multiple spares, to allow multi-fault tolerance (i.e., multiple or repeated failures or damage to the same system).

\section{Design and Support Considerations}

For the implementation of any level of component or board level repair strategy to be successful, systems would need to be designed so that "non-repairable" components were segregated, to the greatest degree possible, on smaller 'daughter' cards, allowing a larger number of smaller cards to be carried. Further, the circuit boards and LRUs must be designed for repair, which includes allowing accessibility to the boards, use of common components when possible, board layouts that reduced the difficulty of performing a repair, common LRU and board interfaces where possible, and extensive BIT to reduce the diagnostic tasks left to a crew member. These techniques will 
reduce the crew workload during a repair, and the amount of training time necessary for crew competence. Ground support teams who are able to examine diagnostic test results (with engineering models of electronics on hand) can greatly aid in the diagnosis and repair of faulty electronics. Finally, one or more crew members should be knowledgeable in the field of electronics. This does not mean that a crew member should be an electronics engineer or technician, but should be trained in some electronics repair similar to the U.S. Navy $2 \mathrm{M}$ program ${ }^{14}$, or similar program focused on the types of repairs and components expected during a space mission.

\section{Conclusions}

This paper examines strategies for the repair of faulty electronics in long duration space missions. The discussion first focused on factors affecting the crew's ability to perform these repairs. These factors include designing the electronics so they can be repaired during a mission, the diagnostics required to both determine the source of a fault and to test a repair before returning the system to use, and crew considerations including training, experience, and ground support through telescience. The paper then discusses three general strategies for performing repairs during a mission: replacing a LRU, repairing components on circuit boards within a faulty LRU, and reprogramming or scavenging parts from a less critical LRU for a faulty, but necessary, LRU. Finally, the authors recommend a mix of replacing entire LRUs when the system is a critical component, having spare circuit boards for circuits requiring complex repair techniques, and spare components for LRUs that are not mission critical and do not require difficult repair procedures. Combined, these methods reduce the volume and mass required for spare parts and increase the capabilities and increase the likelihood of overall mission success.

While the development and implementation of such a repair capability will require effort and expenditure, the cost of failing to provide a well-planned repair capability could be catastrophic, both economically and in human terms, if the failure to make such repairs were to lead to mission failure and loss of life. When considered in those terms, the efforts needed to explore, develop, and implement appropriate repair strategies to allow sustainable and supportable long-duration missions don’t appear nearly as costly.

\section{Acknowledgments}

The authors wish to thank Gary Latta and Andrew Ganster of the Naval Surface Warfare Center, Eric Anderson of Zin Technologies, and Gary Gorecki of NASA Glenn Research Center for their assistance in this effort. This work is funded by the Supportability Project in NASA's Exploration Technology Development Program Office (Barmac Taleghani, Program Manager, NASA Langley Research Center).

\section{References}

1 Beddingfield, K.L., Leach, R.D., and Alexander, M.B. (editor), "Spacecraft System Failures and Anomalies Attributed to the Natural Space Environment”, NASA Reference Publication 1390, August, 1996.

2 Rutledge, P.J., Mosleh, A., "Dependent-Failures in Spacecraft: Root Causes, Coupling Factors, Defenses, and Design Implications”, Proceedings of the IEEE Annual Reliability and Maintainability Symposium, 0149-144X/95, (1995), pp. 337-342.

3 Constellation Architecture Requirements Document, NASA-CxP-70000, effective date September 18, 2006.

4 Accola, A., Fincannon, H.J., Williams, G. J., and Meier, R. T., "Sensitivity Study of Space Station Freedom Operations Cost and Selected User Resources," IAA Symposium on Space Systems Cost Estimation Methodologies and Applications, San Diego, May 1990.

5 Grugel, R.N., Cotton, L.J., Segre, P.N., Ogle, J.A., Funkhouser, G., Parris, F., Murphy, L., Gillies, D., Hua, F., and Anilkumar, A.V., "The In-Space Soldering Investigation (ISSI): Melting and Solidification Experiment Aboard the International Space Station”, 44 ${ }^{\text {th }}$ AIAA Aerospace Sciences Meeting and Exhibit, 2006, AIAA 2006-521.

6 Pettegrew, R.D., Struk, P.M., Watson, J.K., and Haylett, D.R., “Experimental Methods in Reduced Gravity Soldering Research”, NASA-TM 2002-211993 (2002).

7 Pettegrew, R.D., Struk, P.M., Watson, J.K., Haylett, D.R., and Downs, R.S. “Gravitational Effects on Solder Joints”, Welding Journal, pp. 44-48 (2003).

8 Struk, P.M., Pettegrew, R.D., Downs, R.S., and Watson, J.K., "The Effects of an Unsteady Reduced Gravity Environment on the Soldering Process”, AIAA-2004-1311 and NASA / TM -2004-212946 (2004).

9 Struk, P.M., Pettegrew R.D., Downs, R.S., and Watson, J.K., "The Influence Of Gravity on Joint Shape for Through-Hole Soldering”, AIAA-2005-0541 and NASA / TM 2005-213589 (2005).

10 Watson, J.K., Struk, P.M., Pettegrew, R.D., and Downs, R.S., "Experimental Investigation of Solder Joint Defect Formation and Mitigation in Reduced-Gravity Environments”, AIAA Journal of Spacecraft and Rockets, accepted for publication, 2006. 
11 Pettegrew, R.D., Easton, J., Struk, P.M., Anderson, E., “In-Flight Manual Electronics Repair for Deep Space Missions”, IEEEAC Paper \#1208, Submitted for IEEE Conference, March, 2007; also to be a NASA TM, spring, 2007.

12 Combustion Integrated Rack Comprehensive Design Review, FCF-DOC-1421, 2004 (NASA document).

13 Pettegrew, R.D., Easton, J., Struk, P.M., “Repair of Electronics for Long-Duration Spaceflight”, $45^{\text {th }}$ AIAA Aerospace Sciences Meeting and Exhibit, 2007, AIAA 2007-1364.

14 Naval Surface Warfare Crane center information, http://www.crane.navy.mil/2m/2mpage.htm 